\title{
Josephson Current as a Boundary Condition for Gor'kov Equations
}

\author{
Yu. N. Ovchinnikov ${ }^{1,2}(\mathbb{0}$ \\ Received: 14 October 2021 / Accepted: 7 December 2021 / Published online: 29 January 2022 \\ (C) The Author(s) 2022
}

\begin{abstract}
The magnitude of the Josephson current completely determines the value of the order parameters in superconductors separated by a barrier of low transparency, up to the second order in transparency. The second harmonic in the current value is equal to zero in the second approximation of perturbation theory with respect to the barrier transparency, regardless of the approximation within which the bulk superconductors are considered. When considering the correction terms, a new small parameter arises associated with the height of the potential barrier. This effect leads to the suppression of the modulus of the order parameter at the barrier surface and, hence, to the renormalization of the amplitude of the Josephson current.
\end{abstract}

Keywords Josephson effect · Boundary condition · Quasi-classical approximation

\section{Introduction}

In order to describe the current state in a superconductor divided into two parts by a tunnel barrier, it is necessary to have the equations describing the state of superconductors and an expression for the current density in the entire space, including the barrier region. The Gor'kov's [1] equations written for the Green functions in presence the field of a potential barrier of small thickness and low transparency usually are used for this purpose. When the tunneling barrier has a low transparency, the above-mentioned general problem, being considered in the first two orders of the expansion in series by transparency, can be divided into two parts. The first one consists in the choice of the type of approximation for description of the bulk superconductors. The second part dwells in obtaining of the boundary conditions for the corresponding equations and for the current density followed from the Gor'kov equations.

The second part of the problem was already solved in [2] in the second order of perturbation theory in the barrier transparency. The presence of a tunnel barrier with a finite transparency between two superconductors leads to the appearance of Josephson effect [3]. The current density value for the Josephson effect imposes the boundary

Yu. N. Ovchinnikov

ovc@itp.ac.ru

1 Max-Planck Institute for Physics of Complex Systems, Dresden 01187, Germany

2 Landau Institute for Theoretical Physics, RAS, Chernogolovka 142432, Moscow District, Russia condition for the problem under consideration. The law of the current conservation throughout the entire space, including the area inside the barrier, plays a key role in obtaining of this boundary condition. The absence of corrections to the modulus of the order parameter proportional to the current density in the first order of perturbation theory is also an important factor.

For a deeper understanding of the problem under consideration, we will consider four different approximations for describing the bulk superconductors:

- Ginzburg-Landau functional [4]

- generalized Ginzburg-Landau functional [5]

- Gor'kov equations for dirty superconductors

- equations for the Green's functions integrated with respect to the energy variable [6].

\section{Josephson Current in a Low-Transparency Tunnel Junction}

The current density for a tunneling barrier of low transparency was obtained in [2] for a wide range of temperatures, impurity concentrations, and various types of electron reflection from the barrier surface:

$j_{\mathrm{tr}}=\frac{\pi}{e S R}\left|\Delta_{1} \Delta_{2}\right| \sin \left(\phi_{2}-\phi_{1}\right) T \sum_{\omega}\left[\left(\omega^{2}+\left|\Delta_{1}\right|^{2}\right)\left(\omega^{2}+\left|\Delta_{2}\right|^{2}\right)\right]^{-1 \mid 2}$

Here $R$ is the resistance of the tunnel barrier, $S$ is the area of the barrier, $\left\{\left|\Delta_{1,2}\right| ; \phi_{1,2}\right\}$ are the values of the moduli and phases of the order parameters on the surface of the barrier. 
When deriving Eq. (1), the current conservation law in the entire space, including the sub-barrier region, was essentially used.

Equation (1) allows to study the current state of superconductors separated by a tunneling barrier within the perturbation theory in the transparency of the tunneling barrier up to the second order inclusive. The finite value of the tunnel barrier height results in a weak renormalization of the amplitude of the tunneling current in Eq. (1), while its dependence on the phase difference remains unchanged. The details of this effect will be discussed in Sect. 6 .

\section{Approach by the Ginzburg-Landau Functional for the Description of the Bulk Superconductors}

The Ginzburg-Landau functional is usually written as

$$
\begin{aligned}
F_{S}-F_{N}= & \nu \int d^{3} r\left\{\ln \left(\frac{T_{c}}{T}\right)|\Delta|^{2}+\frac{7 \zeta(3)}{16 \pi^{2} T^{2}}|\Delta|^{4}\right. \\
& \left.+\frac{\pi D}{8 T}\left|\left(\frac{\partial}{\partial \mathbf{r}}-2 i e \mathbf{A}\right) \Delta\right|^{2}\right\}+\frac{1}{8 \pi} \int d^{3} r\left(\operatorname{rot} \mathbf{A}-\mathbf{H}_{0}\right)^{2}
\end{aligned}
$$

where $v=m P_{0} / 2 \pi^{2}$ - is the density of electron states at the Fermi surface, $A$ is the vector potential, $\zeta(x)$ is the Riemann zeta function

$D=\frac{\nu l_{\mathrm{tr}}}{3} \eta(T), \quad \eta(T)=1-\frac{8 T \tau_{\mathrm{tr}}}{\pi}\left(\psi\left(\frac{1}{2}+\frac{1}{4 \pi T \tau_{\mathrm{tr}}}\right)-\psi\left(\frac{1}{2}\right)\right)$,

$\mathbf{H}_{0}$ is the external magnetic field, $\psi(k)$ is the Euler psi-function.

Below we restrict ourselves by consideration of a superconductor taken in the form of a wire of a small cross section divided into two parts by a low-transparency tunnel barrier. The current flow does not change the moduli of the order parameter $\left|\Delta_{1,2}\right|$ (this statement is valid in the first order of perturbation theory in the small parameter $j_{\text {tr }} / j_{\text {dep }}<<1$, where $j_{\text {tr }}$ is the tunneling current density and $j_{\text {dep }}$ is the critical depairing current). Hence, the expressions for the order parameters can be represented in the form

$\Delta_{1,2}=\left|\Delta_{1,2}\right| \exp \left(i k_{1,2} z\right) \exp \left(i \phi_{1,2}\right)$

Equation (4) is valid in the whole superconductor up to the surface of the tunnel barrier.

The expression for the current density follows from the Ginzburg-Landau functional

$j=\frac{i \pi e v D}{4 T}\left(\Delta \partial_{+} \Delta^{*}-\Delta^{*} \partial_{-} \Delta\right), \quad \partial_{ \pm}=\frac{\partial}{\partial \mathbf{r}} \pm 2 i e \mathbf{A}$
The current conservation law side by side Eqs. (1), (4) and (5) determines the value of the parameters $\left\{k_{1,2} ; \phi_{2}-\phi_{1}\right\}$ for a given current density

$j_{\text {tr }}=j_{1}=j_{2} ; \quad j_{\text {tr }}=\left(\frac{\pi e v D}{2 T}|\Delta|^{2} k\right)_{1}=\left(\frac{\pi e v D}{2 T}|\Delta|^{2} k\right)_{2}$

In the case when $\left|\Delta_{1}\right|=\left|\Delta_{2}\right|$, the tunneling current density $j_{\text {cr }}$ is equal to [2]:

$j_{\mathrm{tr}}=\frac{\pi|\Delta|}{2 e S R} \operatorname{th}\left(\frac{|\Delta|}{2 T}\right) \sin \left(\phi_{2}-\phi_{1}\right)$

Equations (6) and (7) demonstrate that the tunneling current does not contain the second harmonic of the order parameters phase difference $\phi_{2}-\phi_{1}$. Let us recall that this statement is valid with the accuracy up to the second order of perturbation theory in the barrier transparency.

Let us now consider a more general case in which for description of the bulk superconductor one use the generalized Ginzburg-Landau functional [5]

$$
\begin{aligned}
F_{S}-F_{N}= & v \int d^{3} r\left\{-\ln \left(\frac{T_{c}}{T}\right)|\Delta|^{2}+\frac{7 \zeta(3)}{16 \pi^{2} T^{2}}|\Delta|^{4}\right. \\
& +\frac{\pi D}{8 T}\left|\partial_{-} \Delta\right|^{2}-\frac{31 \zeta(5)|\Delta|^{6}}{128 \pi^{4} T^{4}}-\frac{v^{4}\left(1 / \tau-1 / \tau_{1}\right)}{288} a_{1}\left|\partial_{-}^{2} \Delta\right|^{2} \\
& -\frac{v^{2}}{24} a_{2}|\Delta|^{2}\left|\partial_{-} \Delta\right|^{2}-\frac{v^{2}}{24} b_{2}\left(\frac{1}{2}\left(\frac{\partial|\Delta|^{2}}{\partial r}\right)^{2}+2|\Delta|^{2}\left|\partial_{-} \Delta\right|^{2}\right) \\
& -\frac{v^{4}}{80} b_{1}\left(\left(\left|\partial_{-}^{2} \Delta\right|^{2}+4 e^{2} \mathbf{H}^{2}|\Delta|^{2}\right)-\frac{2 i e}{3} \operatorname{rot} \mathbf{H}\right. \\
& \left.\left.\cdot\left(\Delta^{*} \partial_{-} \Delta-\Delta \partial_{+} \Delta^{*}\right)\right)\right\}+\frac{1}{8 \pi} \int d^{3} r\left(\operatorname{rot} \mathbf{A}-\mathbf{H}_{0}\right)^{2}
\end{aligned}
$$

In Eq. (8) $v$ - velocity on the Fermy surface,

$$
\begin{aligned}
& b_{2}=2 \pi T \sum_{\omega>0} \frac{1}{\omega^{4}\left(\omega+1 / 2 \tau_{\mathrm{tr}}\right)}, \\
& a_{2}=2 \pi T \sum_{\omega>0} \frac{1}{\omega^{3}\left(\omega+1 / \tau_{\mathrm{tr}}\right)^{2}}, \\
& b_{1}=2 \pi T \sum_{\omega>0} \frac{1}{\omega^{2}\left(\omega+1 / 2 \tau_{\mathrm{tr}}\right)^{2}\left(\omega+\frac{1}{2}\left(1 / \tau-1 / \tau_{2}\right)\right)}, \\
& a_{1}=2 \pi T \sum_{\omega>0} \frac{1}{\omega^{3}\left(\omega+1 / 2 \tau_{\mathrm{tr}}\right)^{2}\left(\omega+\frac{1}{2}\left(1 / \tau-1 / \tau_{2}\right)\right)}
\end{aligned}
$$

The summation over Matsubara frequencies in Eq. (9) was performed in Ref. [5].

As above, the expressions for order parameters $\Delta_{1,2}$ that are valid up to the barrier surface can be chosen in the form of Eq. (4).

Using the Ginzburg-Landau functional in the form of Eq. (8) one can obtain the following expression for the current density 


$$
\begin{aligned}
j= & \frac{i \pi e v D}{4 T}\left(\Delta \partial_{+} \Delta^{*}-\Delta^{*} \partial_{-} \Delta\right)-\frac{i e v v^{4}}{4}\left\{\frac{1}{36}\left(1 / \tau-1 \tau_{2}\right) a_{1}+\frac{1}{10} b_{1}\right\} \\
& \times\left\{\left(\left(\partial_{-} \Delta\right)\left(\partial_{+}^{2} \Delta^{*}\right)-\left(\partial_{+} \Delta^{*}\right)\left(\partial_{-}^{2} \Delta\right)\right)+\left(\Delta^{*} \partial_{-}^{3} \Delta-\Delta \partial_{+}^{3} \Delta^{*}\right)\right\} \\
& -\frac{i e v v^{2}}{12}\left(a_{1}+2 b_{2}\right)\left(\Delta \partial_{+} \Delta^{*}-\Delta^{*} \partial_{-} \Delta\right)|\Delta|^{2} \\
& -\frac{i e v v^{4} b_{1}}{120}\left(-4 i e|\Delta|^{2} \operatorname{rot} \mathbf{H}+\operatorname{rotrot}\left(\Delta^{*} \partial_{-} \Delta-\Delta \partial_{+} \Delta^{*}\right)\right) \\
& +\frac{e^{2} v v^{4} b_{1}}{10} \operatorname{rot}\left(|\Delta|^{2} \operatorname{rot} \mathbf{A}\right)
\end{aligned}
$$

Substituting the order parameters $\Delta_{1,2}$ in the form of Eq. (4) into Eq. (10), one obtains the expression for the value of the current density in superconductors $[1,2]$

$$
\begin{aligned}
j_{1,2}= & \left\{\frac{\pi e v D}{2 T}|\Delta|^{2} k-\frac{e v^{4} k^{3}}{2}\left(\frac{1}{18}\left(1 / \tau-1 / \tau_{2}\right) a_{1}+\frac{1}{5} b_{1}\right)|\Delta|^{2}\right. \\
& \left.-\frac{e v v^{2}}{6}\left(a_{2}+2 b_{2}\right)|\Delta|^{4} k\right\}_{1,2}
\end{aligned}
$$

Finally, the current conservation law

$j_{\mathrm{tr}}=j_{1}=j_{2}$

determines the value of the quantities $\left\{k_{1}, k_{2}, \phi_{2}-\phi_{1}\right\}$. From Eq. (12) it follows that in the adopted approximation of the second order of the perturbation theory with respect to the transparency of the barrier, the second harmonic in the current density is absent.

\section{Superconductor with Short Electron Mean Free Path}

In a superconductor with short electron mean free path ("dirty" superconductor), the Gor'kov equations can be reduced to a system of differential equations for the Green's functions, integrated over the energy variable and averaged over the directions of the momentum on the Fermi surface [6]

$$
\begin{aligned}
& \tau_{z} \frac{\partial G}{\partial \tau}+\frac{\partial G}{\partial \tau^{\prime}} \tau_{z}+[e \phi-\hat{\Delta}, G]= \\
& -i D\left[\frac{\partial}{\partial \mathbf{R}}-i e \mathbf{A} \tau_{z}, G\left(\frac{\partial}{\partial \mathbf{R}}-i e \mathbf{A} \tau_{z}\right) G\right] \\
& +\left(i / 2 \tau_{S}\right)\left[\tau_{z}, G \tau_{z} G\right]+e D \tau_{z} \delta\left(\tau-\tau^{\prime}\right) \operatorname{div} \mathbf{A}
\end{aligned}
$$

$$
\int_{0}^{1 / T} G\left(\tau, \tau_{1}, \mathbf{R}\right) G\left(\tau_{1}, \tau^{\prime}, \mathbf{R}\right) d \tau_{1}=\delta\left(\tau-\tau^{\prime}\right)
$$

where

$\hat{\Delta}=\left(\begin{array}{cc}0 ; & \Delta \\ -\tilde{\Delta} ; & 0\end{array}\right) ; \quad G=\left(\begin{array}{cc}g ; & F \\ -\tilde{F} ; & \tilde{g}\end{array}\right) ; \quad \Delta=i|\lambda| \nu F(\tau, \tau, \mathbf{R})$
Here $\phi$ is the scalar potential, [,] is a commutator, $\tau_{S}$ is electron scattering time with the spin flip. In its turn the current density is determined by the expression

$$
\begin{aligned}
j(\tau)= & \pi \sigma_{0}\left\{-\mathbf{A}(\tau) \delta\left(\tau-\tau^{\prime}\right)\right. \\
& \left.+\frac{i}{2 e} \operatorname{Sp} \tau_{z} \int_{0}^{1 / \tau} G\left(\tau, \tau_{1}\right)\left(\frac{\partial}{\partial \mathbf{R}}-i e \mathbf{A}\left(\tau_{1}\right) \tau_{z}\right) G\left(\tau_{1}, \tau^{\prime}\right) d \tau_{1}\right\}_{\tau=\tau^{\prime}}
\end{aligned}
$$

where $\sigma_{0}=e^{2} p^{2} l_{t r} / 3 \pi^{2}$ is the conductivity of the metal in its normal state. The expression for the order parameters $\Delta_{1,2}$ which is valid up to the surface of the tunnel barrier, can be again represented in the form of Eq. (4) with constant $\Delta_{1,2}$.

The matrix Green's function $G$ we will seek in the form

$F=-\beta \exp (i(k z+\phi)), \quad \tilde{F}=-F^{*}, \quad g=\alpha, \quad \tilde{g}=-\alpha$

where the parameters $\{\phi, \alpha, \beta\}$ are independent on coordinate constants.

Substituting Eq. (16) into Eqs. (13)-(15), we obtain two equations for $\{\alpha, \beta\}$ and the expression for the current density:

$\alpha^{2}(\omega)+\beta^{2}(\omega)=1 ; \quad \alpha(\omega) \Delta-\beta(\omega) \omega=\alpha(\omega) \beta(\omega) D k^{2} / 2$

$j_{1,2}=\left\{\frac{\pi \sigma_{0}}{e} k \cdot T \sum_{\omega} \beta^{2}(\omega)\right\}_{1,2}$

The quantities $\left\{k_{1,2}\right\}$ are solutions of the equation

$j_{1,2}=j_{\text {tr }}$

Analysis of Eqs. (17)-(19), in the frameworks of the perturbation theory up to the second order with respect to the barrier transparency, demonstrates the absence of the second harmonic of the phase difference $\phi_{2}-\phi_{1}$ in the tunneling current density.

\section{Quasi-Classical Approximation Based on the Integrated Over the Energy Variable Green Functions Formalism}

The equation for the Green's functions integrated over the energy variable can be written in the form [6]

$$
\begin{aligned}
& \frac{1}{m}\left(\mathbf{P} \frac{\partial G}{\partial \mathbf{R}}\right)+i \tau_{z} \frac{\partial G}{\partial \tau}+i \frac{\partial G}{\partial \tau^{\prime}} \tau_{z} \\
& +\left[-\frac{i e}{m}(\mathbf{P A}) \tau_{z}-i \hat{\Delta}+i e \phi+i n \hat{\Sigma}, G\right]=0, \\
& \int_{0}^{1 / T} G_{\mathbf{P}}\left(\tau, \tau_{1}, \mathbf{R}\right) G_{\mathbf{P}}\left(\tau_{1}, \tau^{\prime}, \mathbf{R}\right) d \tau_{1}=\delta\left(\tau-\tau^{\prime}\right)
\end{aligned}
$$


while the current

$j=-\frac{i e v}{4 m} \int d \Omega_{\mathbf{P}} \mathbf{P S p}\left(\tau_{z} G_{\mathbf{P}}\right)$

Here $n$ is the impurity concentration, $\phi$ is the scalar, and A is the vector potentials. The self-energy part $\Sigma$ in the Born approximation is equal to

$\hat{\Sigma}_{\mathbf{P P}}=-\frac{i \nu}{2} \int \sigma_{\mathbf{P P}^{\prime}} G_{\mathbf{P}^{\prime}} d \Omega_{\mathbf{P}^{\prime}}$

with $\sigma_{\mathbf{P P}^{\prime}}$ as the electron scattering cross section of electron by impurity.

In the geometry under consideration, the expression for the order parameter $\Delta$, which is valid up to the barrier surface, can be chosen in the form

$\hat{\Delta}=\left(\begin{array}{cc}0 ; & |\Delta| e^{i k z+i \phi} \\ -|\Delta| e^{-i k z-i \phi} ; & 0\end{array}\right)$

where the values $\{|\Delta|, k, \phi\}$ do not depend on the coordinates. The matrix Green's function $G$ acquires the form

$G=\left(\begin{array}{c}\alpha_{\mathbf{P}} ;-i \beta_{\mathbf{P}} e^{i k z+i \phi} \\ i \tilde{\beta}_{\mathbf{P e}}^{-i k z-i \phi} ;-\alpha_{\mathbf{P}}\end{array}\right)$

Substitution of Eq. (24) into Eqs. (20)-(21) results in the following relationships for the quantities $\{\alpha, \beta, \tilde{\beta}, k, \phi\}$

$\tilde{\beta}_{\mathbf{P}}=\beta_{\mathbf{P}} ;$

$\alpha_{\mathbf{P}}^{2}(\omega)+\beta_{\mathbf{P}}(\omega) \tilde{\beta}_{\mathbf{P}}(\omega)=1 ;$

$\alpha_{\mathbf{P}}(\omega)|\Delta|-\omega \beta_{\mathbf{P}}(\omega)+\frac{n v}{2}$

$\int d \Omega_{\mathbf{P}^{\prime}} \sigma_{\mathbf{P P}^{\prime}}\left(\alpha_{\mathbf{P}} \beta_{\mathbf{P}^{\prime}}-\alpha_{\mathbf{P}^{\prime}} \beta_{\mathbf{P}}\right)-\frac{i}{2}(\mathbf{v k}) \beta_{\mathbf{P}}(\omega)=0$

As the small parameter here appears the ratio of the tunneling current density to that one of the depairing current. The second-order correction with respect to this parameter does not appear in the current density determined by Eq. (21). Consequently, the second harmonic of the phase difference $\phi_{2}-\phi_{1}$ is absent in the tunneling current density also under this, fairly general, assumptions made in this section.

In the first order in the barrier transparency analyzing Eq. (25), one finds the following expression for the quantities $\left\{\alpha_{\mathbf{P}}, \beta_{\mathbf{P}}\right\}$

$$
\begin{aligned}
\alpha_{\mathbf{P}} & =\frac{\omega}{\left(\omega^{2}+|\Delta|^{2}\right)^{1 / 2}}+\alpha_{\mathbf{P}}^{(1)} ; \\
\beta_{\mathbf{P}} & =\frac{|\Delta|}{\left(\omega^{2}+|\Delta|^{2}\right)^{1 / 2}}-\frac{\omega}{|\Delta|} \alpha_{\mathbf{P}}^{(1)} ; \\
\alpha_{\mathbf{P}}^{(1)} & =\frac{i}{2} \frac{|\Delta|^{2}}{\omega^{2}+|\Delta|^{2}} \frac{(\mathbf{v k})}{\sqrt{\omega^{2}+|\Delta|^{2}}+1 / 2 \tau_{\mathrm{tr}}}
\end{aligned}
$$

Substituting expressions (26) into Eq. (21) for the current density, one finds the values of the parameters determining the current state in the structure under consideration:

$j_{1,2}=\left\{\frac{\pi e v P \mathbf{k}}{3 m} T \sum_{\omega} \frac{|\Delta|^{2}}{\sqrt{\omega^{2}+|\Delta|^{2}}+1 / \tau_{\mathrm{tr}}}\right\}, \quad j_{\mathrm{tr}}=j_{1}=j_{2}$

One sees that the second harmonic of the phase difference $\phi_{2}-\phi_{1}$ again does not appear in the tunneling current even in the considered most general case.

\section{The Role of the Finite Value of the Barrier Height}

The finiteness of the tunnel barrier height results in the renormalization of the order parameter magnitude in the vicinity of the barrier even in the absence of flowing current. Analysis of the Gor'kov's equation when current is zero leads to the following expression for the Green's function $F$ inside the barrier

$$
\begin{aligned}
F(\omega, z, z)= & C_{1} \exp \left(-2\left(\frac{d}{2}+z\right) \sqrt{2 m_{1} V}\right) \\
& +C_{2} \exp \left(2\left(-\frac{d}{2}+z\right) \sqrt{2 m_{2} V}\right)
\end{aligned}
$$

where $C_{1,2}$ are some constants, $d$ is the thickness of the barrier, $V$ is the height of the barrier. A significant jump of the function $F$ occurs at the surface of the barrier when its transparency is small. As result, instead of the standard requirement at the barrier surface

$\frac{\partial|\Delta|}{\partial z}=\left.0\right|_{z= \pm d / 2}$

one obtains the following boundary condition for the function $\beta_{P}(\omega)$

$\left\{\left(\mathbf{n} \frac{\partial}{\partial \mathbf{r}}\right) \ln <\beta_{\mathbf{P}}(\omega)>=-\frac{4}{\left(1+2 m V / p^{2}\right)} \frac{\sqrt{2 m V}}{(p / \kappa)^{2}}\right.$

where $\mathbf{n}$ is the normal to the superconductor surface, $\kappa^{-1}$ correlation length definite in Eq. (39) and

$<\beta_{\mathbf{P}}(\omega)>=\frac{1}{4 \pi} \int d \Omega_{\mathbf{P}} \beta_{\mathbf{P}}(\omega)$

(see Eqs. (20) and (24))

Right-hand side of Eq. (30) is the first nonzero term of expansion over steps of inverse quasiclassical parameter.

In the case under consideration, the system of Eq. (20) is reduced to the form 


$$
\begin{aligned}
\left(\mathbf{v} \frac{\partial}{\partial \mathbf{r}}\right) \beta_{\mathbf{P}}+2 \omega \beta_{\mathbf{P}}-2|\Delta| \alpha_{\mathbf{P}}+n v \int d \Omega_{\mathbf{P}^{\prime}} \sigma_{\mathbf{P P}^{\prime}}\left(\beta_{\mathbf{P}} \alpha_{\mathbf{P}^{\prime}}-\alpha_{\mathbf{P}} \beta_{\mathbf{P}^{\prime}}\right) & =0 ; \\
-\left(\mathbf{v} \frac{\partial}{\partial r} \tilde{\beta}_{\mathbf{P}}+2 \omega \tilde{\beta}_{\mathbf{P}}-2|\Delta| \alpha_{\mathbf{P}}+n v \int d \Omega_{\mathbf{P}^{\prime}} \sigma_{\mathbf{P P}^{\prime}}\left(\tilde{\beta}_{\mathbf{P}} \alpha_{\mathbf{P}^{\prime}}-\alpha_{\mathbf{P}} \tilde{\beta}_{\mathbf{P}^{\prime}}\right)\right. & =0 ; \\
\left(\mathbf{v} \frac{\partial}{\partial \mathbf{r}}\right) \alpha_{\mathbf{P}}+|\Delta|\left(\tilde{\beta}_{\mathbf{P}}-\beta_{\mathbf{P}}\right)+\frac{n v}{2} \int d \Omega_{\mathbf{P}^{\prime}} \sigma_{\mathbf{P P}^{\prime}}\left(\beta_{\mathbf{P}^{\prime}} \tilde{\beta}_{\mathbf{P}}-\beta_{\mathbf{P}} \tilde{\beta}_{\mathbf{P}^{\prime}}\right) & =0 ; \\
\alpha_{\mathbf{P}}^{2}(\omega)+\beta_{\mathbf{P}}(\omega) \tilde{\beta}_{\mathbf{P}}(\omega) & =1
\end{aligned}
$$

$|\Delta| \ln \left(\frac{T_{c}}{T}\right)=2 \pi T \sum_{\omega>0}\left(\frac{|\Delta|}{\omega}-<\beta_{\mathbf{P}}(\omega)>\right)$

We look for solution of this system of equations by means of the method of perturbation theory with respect to the small parameter determined by Eq. (30). In result one finds

$$
\begin{aligned}
& \beta_{\mathbf{P}}(\omega)=\frac{\left|\Delta_{0}\right|}{\left(\omega^{2}+|\Delta|^{2}\right)^{1 / 2}}+\delta \beta^{(1)}(\omega)-\left(\mathbf{v} \frac{\partial}{\partial r}\right) \delta \beta^{(2)}(\omega) ; \\
& \tilde{\beta}_{\mathbf{P}}(\omega)=\frac{\left|\Delta_{0}\right|}{\left(\omega^{2}+|\Delta|^{2}\right)^{1 / 2}}+\delta \beta^{(1)}(\omega)-\left(\mathbf{v} \frac{\partial}{\partial \mathbf{r}}\right) \delta \beta^{(2)}(\omega) ; \\
& \alpha_{\mathbf{P}}(\omega)=\frac{\omega}{\left(\omega^{2}+\left|\Delta_{0}\right|^{2}\right)^{1 / 2}}-\frac{\left|\Delta_{0}\right| \delta \beta^{(1)}(\omega)}{\omega}
\end{aligned}
$$

where

$$
\delta \beta^{(2)}(\omega)=-\frac{1}{2 \omega\left(1+\frac{1}{2 \tau_{\mathrm{tr}}\left(\omega^{2}+\left|\Delta_{0}\right|^{2}\right)^{1 / 2}}\right)} \delta \beta^{(1)}(\omega),
$$

while $\Delta_{0}$ is the value of the order parameter modulus at large distances from the barrier. The function $\delta \beta^{(1)}(\omega)$ satisfies the boundary condition (30) and the following equation

$$
\begin{aligned}
& -\frac{v^{2}}{12 \omega\left(1+\frac{1}{2 \tau_{\mathrm{tr}}\left(\omega^{2}+\left|\Delta_{0}\right|^{2}\right)^{1 / 2}}\right)} \frac{\partial^{2}}{\partial z^{2}} \delta \beta^{(1)}+\frac{\omega^{2}+\left|\Delta_{0}\right|^{2}}{\omega} \delta \beta^{(1)}(\omega) \\
& =\Delta^{(1)} \frac{\omega}{\left(\omega^{2}+\left|\Delta_{0}\right|^{2}\right)^{1 / 2}}
\end{aligned}
$$

where

$\Delta=\Delta_{0}+\Delta^{(1)}$

The functions $\left\{\Delta^{(1)}, \beta^{(1)}(\omega)\right\}_{1,2}$ can be chosen in the form $\Delta_{1,2}^{(1)}=\gamma_{1,2} \exp \left(-\kappa_{1,2}|z|\right), \quad\left\{\delta \beta_{(1)}(\omega)=\Gamma(\omega) \exp (-\kappa|z|)\right\}_{1,2}$ $\left\{\Gamma(\omega)=\frac{\gamma \omega^{2}}{\left(\omega^{2}+\left|\Delta_{0}\right|^{2}\right)^{3 / 2}} /\left(1-\frac{\kappa^{2} v^{2}}{12\left(\omega^{2}+|\Delta|^{2}\right)}\right.\right.$ $\left.\left.\left(1+\frac{1}{2 \tau_{\mathrm{tr}}\left(\omega^{2}+\left|\Delta_{0}\right|^{2}\right)^{1 / 2}}\right)^{-1}\right)\right\}_{1,2}$

$$
\left\{\Gamma(\omega)=-\frac{4 \sqrt{2 m V}}{\left.\kappa\left(1+2 m V / p^{2}\right)(p / \kappa)^{2}\right)} \cdot \frac{\left|\Delta_{0}\right|}{\left(\omega^{2}+\left|\Delta_{0}\right|^{2}\right)^{1 / 2}}\right\}_{1,2}
$$

Substituting the expressions for the quantities $\left\{\Delta^{(1)}, \Gamma(\omega)\right\}$ into Eq. (33), one obtains the equation for the quantity $\kappa$ in explicit form

$$
\begin{gathered}
\left\{2 \pi T \sum _ { \omega } \left[\frac{1}{\left(\omega^{2}+\left|\Delta_{o}\right|^{2}\right)^{1 / 2}}-\frac{\omega^{2}}{\left(\omega^{2}+\left|\Delta_{0}\right|^{2}\right)^{3 / 2}} /\right.\right. \\
\left.\left.\left(1-\frac{\kappa^{2} v^{2}}{12\left(\omega^{2}+\left|\Delta_{0}\right|^{2}\right)}\left(1+\frac{1}{2 \tau_{\mathrm{tr}}\left(\omega^{2}+\left|\Delta_{0}\right|^{2}\right)^{1 / 2}}\right)^{-1}\right)\right]\right\}_{1,2}
\end{gathered}
$$

In the presence of non-zero current the Green's function $G$ can be chosen in the form

$G=\left(\begin{array}{cc}\alpha_{\mathbf{P}} ; & -i \beta_{\mathbf{P e}}^{i \chi(z)+i \phi} \\ i \tilde{\beta}_{\mathbf{P e}}^{-i \chi(z)-i \phi} ; & -\alpha_{\mathbf{P}}\end{array}\right)$

The phase $\chi(z)$ is equal zero at the surface of the barrier. The quantities $\left\{\alpha_{\mathbf{P}},<\beta_{\mathbf{P}}>,<\tilde{\beta}_{\mathbf{P}}>, \Delta\right\}$ can be taken from Eq. (38), since the corrections to all of them are equal to zero in the linear approximation in current. Normalization condition (20) yields the important equation

$\alpha_{\mathbf{P}}^{2}+\beta_{\mathbf{P}}(\omega) \tilde{\beta}_{\mathbf{P}}(\omega)=1$

Presence of the current leads to emergence of nonzero to the quantities $\left\{\left(\delta \beta_{\mathbf{P}}(\omega)\right)_{\text {cur }},\left(\delta \tilde{\beta}_{\mathbf{P}}(\omega)\right)_{\text {cur }},\left(\delta \alpha_{\mathbf{P}}(\omega)\right)_{\text {cur }}\right\}$, all of them being proportional to the same function $\left(\mathbf{v} \frac{\partial}{\partial \mathbf{r}}\right) \chi$ Eqs. (20) and (40) yield for these corrections

$$
\begin{aligned}
& \left(\delta \tilde{\beta}_{\mathbf{P}}(\omega)\right)_{\mathrm{cur}}=\left(\delta \beta_{\mathbf{P}}(\omega)\right)_{\mathrm{cur}}, \\
& \left(\delta \beta_{\mathbf{P}}(\omega)\right)_{\mathrm{cur}}=-\frac{\left\langle\alpha_{\mathbf{P}}(\omega)>\right.}{\left\langle\beta_{\mathbf{P}}(\omega)>\right.}\left(\delta \alpha_{\mathbf{P}}(\omega)\right)_{\mathrm{cur}}, \\
& \left.\left(\delta \alpha_{\mathbf{P}}(\omega)\right)_{\mathrm{cur}}=\frac{i}{2}<\beta_{\mathbf{P}}(\omega)\right\rangle^{2} \\
& \left(\mathbf{v} \frac{\partial \chi}{\partial \mathbf{r}}\right) \frac{1}{\omega<\alpha_{\mathbf{P}}(\omega)>+|\Delta|<\beta_{\mathbf{P}}(\omega)>+\frac{1}{2 \tau_{\mathrm{tr}}}}
\end{aligned}
$$

Equations (20) and (42) make it possible to obtain the following expression for the current density

$\left\{j=\frac{\pi e v v^{2}}{3} \frac{\partial \chi}{\partial \mathbf{r}} T \sum_{\omega}<\beta_{\mathbf{P}}>^{2} \frac{1}{\omega<\alpha_{\mathbf{P}}(\omega)>+|\Delta|<\beta_{\mathbf{P}}(\omega)>+\frac{1}{2 \tau_{\text {II }}}}\right\}_{1,2}$

The current conservation law transforms Eq. (43) into an equation for the phase $\chi(z)$, valid in whole superconductor up to the barrier. Following Ref. [2], one finds the corrected equation for the Josephson current density in the form

$j_{\mathrm{tr}}=\frac{\pi}{e S R} \sin \left(\phi_{2}-\phi_{1}\right) T \sum_{\omega}<\beta_{\mathbf{P}}(\omega)>_{1}<\beta_{\mathbf{P}}(\omega)>_{2}$ 
In Eq. (44), the quantities $\left\{\left\langle\beta_{\mathbf{P}}(\omega)\right\rangle_{1,2}\right\}$ should be taken at the barrier surface. The current conservation law means that

$j_{\mathrm{tr}}=j_{1}=j_{2}$

\section{Conclusion}

A superconducting current can flow in a superconductor divided into two parts by a barrier (Josephson effect). The correction to the modulus of the order parameter is equal to zero in the first order of the perturbation theory in the value of the tunneling current. An important fact which was found above is the existence of the phase difference jump $\left(\phi_{2}-\phi_{1}\right)$ of the order parameter between the opposite sides of the barrier while maintaining an unambiguous interpretation of the Gor'kov's equation for the function $F$ at the coinciding points in the underbarrier region. As a result, at low transparency of the tunneling barrier, the study of the Josephson effect splits into two parts, as was done above. Knowledge of the solutions of the Schrödinger equation for wave functions in the field of a potential barrier is insufficient to recover the Gor'kov's correlation functions. The boundary conditions obtained on this basis and the results following from them are erroneous [7-9].

In the case of the finite value of the barrier height the derivative from the order parameter on the barrier surface $\left(\mathbf{n} \frac{\partial}{\partial r}\right)|\Delta|$ is non zero.

It is very important that in the first order of perturbation theory in the transparency of the barrier, this slope does not depend on the magnitude of the current density. As a result, the phases $\phi_{1,2}$ can be easily reconstructed using Eq. (43) and they do not acquire additional dependence on the phase difference $\phi_{2}-\phi_{1}$ (see Eqs. (43)-(44)). As a result, the second harmonic in the current density, being calculated in the second order of the perturbation theory with respect to the barrier transparency, turns out to be zero.

The finiteness of the potential barrier height leads to suppression of the order parameter modulus in the vicinity of the barrier and, thus, to a weak renormalization of the Josephson current amplitude. Its phase dependence is of particular interest because in the low-frequency limit the expression for current is retained with the replacement $\partial \Phi / \partial t=2 e V$ ( $V$ is the potential difference across the barrier) [10].

For typical set of junction parameters $\left\{v_{F}=10^{8} \mathrm{sm} / \mathrm{sec}\right.$, $\left.V / \epsilon_{F}=10 ; p / \hbar \kappa=10^{4}, m=9.8 \cdot 10^{-28} \mathrm{gr}\right\} \quad$ we obtain the following smallness value, connected with Eq. (30): $\Delta_{1} / \Delta_{0} \approx 1.15 \cdot 10^{-4}$

Acknowledgements I thanks prof. A. A. Varlamov for assistance by manuscript preparing for publishing and helpful notes.

Funding Open Access funding enabled and organized by Projekt DEAL.

Open Access This article is licensed under a Creative Commons Attribution 4.0 International License, which permits use, sharing, adaptation, distribution and reproduction in any medium or format, as long as you give appropriate credit to the original author(s) and the source, provide a link to the Creative Commons licence, and indicate if changes were made. The images or other third party material in this article are included in the article's Creative Commons licence, unless indicated otherwise in a credit line to the material. If material is not included in the article's Creative Commons licence and your intended use is not permitted by statutory regulation or exceeds the permitted use, you will need to obtain permission directly from the copyright holder. To view a copy of this licence, visit http://creativecommons.org/licenses/by/4.0/.

\section{References}

1. Gor'kov, L.P.: Zh. Eksp. Teor. Fis. 34, 735 (1958) Sov. Phys. JETP 7, 505 (1958)

2. Larkin, A.I., Ovchinnikov, Y.N., Fedorov, M.A.: Zh. Eksp. Teor. Fis. 51, 683 (1966) Sov. Phys. JETP 24, 452 (1967)

3. Josephson, B.: Phys. Lett. 1, 251 (1962)

4. Ginzburg, V.L., Landau, L.D.: Zh. Eksp. Teor. Fis. 20, 1064 (1950) Collected papers of L. D. Landau, Gordon and Breach, New York 1965, pp. 546-568

5. Ovchinnikov, Y.N.: Zh. Eksp. Teor. Fis. 115, 726 (1999) Sov. Phys. JETP 88, 398 (1999)

6. Larkin, A.I., Ovchinnikov, Y.N.: J. Low Temp. Phys. 10, 407 (1973)

7. Zaitsev, A.V.: Zh. Eksp. Teor. Fis. 86, 1742 (1984) Sov. Phys. JETP 59, 1015 (1984)

8. Kuprianov, M.Y., Lukichev, V.F.: Zh. Eksp. Teor. Fis. 94, 139 (1988) Sov. Phys. JETP 67, 1163 (1988)

9. Osin, A.S., Fominov, Y.V.: Zh. Eksp. Teor. Fis. (2021) arXiv:2105.05786v1 [cond-mat.supr-con]

10. Larkin, A.I., Ovchinnikov, Y.N.: Zh. Eksp. Teor. Fis. 51, 1535 (1966) Sov. Phys. JETP 24, 1071 (1967)

Publisher's Note Springer Nature remains neutral with regard to jurisdictional claims in published maps and institutional affiliations. 